\title{
Index of Retained Strength and Weight Loss on Flexible Pavement Ac-Wc and Hrs-Wc Using Polymer Products Ethylene-Vinyl-Acetate (EVA) and Styrene- Butadiene-Styrene (SBS)
}

\author{
Mirka Pataras ${ }^{1, *}$ Edi Kadarsa ${ }^{1}$ Debby Permata $Y^{1}$ Shara Khairunnisa ${ }^{1}$ \\ M. Alief Akbar ${ }^{1}$ Yondhika Pratama $^{1}$ \\ ${ }^{1}$ Civil Engineering Department, University of Sriwijaya, \\ Jl. Raya Palembang-Prabumulih KM. 32, Inderalaya, Ogan Ilir 30662, Indonesia \\ *Corresponding author.Email: mirkapataras@ft.unsri.ac.id; patarasmirka@gmail.com
}

\begin{abstract}
Road pavements in Indonesia often uses a mixture of Asphalt Concrete (AC) and Hot Rolled Sheet (HRS), which frequently occur premature damage to the pavement caused by extreme climate change, and also rapid development of land transportation causing increased loads on the pavement, causing queues of vehicles that make traffic jams. Over loading that is too long due to queuing of vehicles causes the performance of the asphalt to decrease. To improve and repair the performance of the asphalt, additives were added, namely Styrene Butadiene Styrene (SBS) polymer and Ethylene Vinyl Acetate (EVA) polymer into a mixture of Asphalt Concrete-Wearing Course (AC-WC) and Hot Rolled Sheet-Wearing Course (HRS-WC) using Marshall Method Test, Marshall Immersion Test and Cantabro Test. This research method is in the form of laboratory experiments with material testing and direct observation. Based on the General Specifications of Bina Marga Division 6 Revision 1 of 2018 for Marshall Tests Parameters, the best stability value for the Asphalt Concrete-Wearing Course (AC-WC) was obtained with the addition of SBS polymer rather than other mix. The Index of Retained Strength (IRS) results from the Marshall Immersion test have met the requirements greater than $90 \%$. The percent of Weight loss from the Cantabro Test has met the requirements of less than $20 \%$. Based on the two tests that have been carried out, sample of SBS modified asphalt mixture is better than the standard asphalt mixture and EVA modified asphalt mixture. The test specimen for the mixture of SBS modified asphalt AC-WC produces the highest Index of Retained Strength value, which is $93.33 \%$ compared to the test specimen for the mixture of standard asphalt AC-WC at $92.37 \%$ and the mixture for EVA modified asphalt AC-WC at 92.74\%. The test specimen of SBS modified asphalt HRS-WC mixture also produced the highest Index of Retained Strength of $91.48 \%$ compared to the standard asphalt HRS-WC mixture test specimen of $90.25 \%$ and EVA modified asphalt HRS-WC of $90.58 \%$. On Weight Loss, the specimens of standard asphalt AC-WC mixture are $4.04 \%$, SBS modified AC-WC asphalt mixtures are 3.14\%, and EVA modified AC-WC asphalt mixtures are $3.57 \%$. The SBS modified AC-WC asphalt mixture was better Weight Loss than the standard AC-WC mixture and the EVA modified AC-WC mixture. On Weight Loss, the specimen of standard asphalt HRS-WC mixture was 3.91\%, SBS modified asphalt HRS-WC mixture was $2.86 \%$, and EVA modified asphalt HRS-WC mixture was $3.34 \%$. The SBS modified asphalt HRS-WC mixture performed better Weight Loss than the standard asphalt HRS-WC mixture and the EVA modified asphalt HR-WC mixture.
\end{abstract}

Keywords: Iindex of retained strenght, Ac-Wc and Hrs-Wc 


\section{INTRODUCTION}

In general, road pavements in Indonesia often suffer premature damage, cracking of Asphalt Concrete and rutting of thin layers of asphalt concrete (Hot Rolled Sheet). The main cause of road damage is the reduced and worsening resistance of the pavement layer in accepting heavy traffic which is often associated with durability, resistance to rutting grooves, damage to pavement structures and high wear of surface texture (Dahlan, 1997) [1]. From these problems, research was carried out with the addition of Styrene-ButadieneStyrene (SBS) and Ethylene-Vinyl-Acetate (EVA) polymers into the asphalt in the Asphalt ConcreteWearing Course (AC-WC) mixture and the Hot Rolled Sheet-Wearing Course (HRS-WC) mixture which is expected to improve the performance of the asphalt. This study aims to determine the parameters of the Marshall Test, Marshall Immersion Test and Cantabro Test with reference to the General Specifications of Bina Marga Division 6, Revision 1 of 2018 [2].

Mixture of Asphalt Concrete layers (AC) is divided into three types of mixtures, namely $\mathrm{AC}$ Wearing Course (AC-WC), AC Binder Course (AC$\mathrm{BC}$ ), and $\mathrm{AC}$ Base. Where each maximum particle size of the aggregate for each mixture is $19 \mathrm{~mm}, 25.4 \mathrm{~mm}$, and $37.5 \mathrm{~mm}$. The Laston or AC mixture which softens the polymer asphalt material is referred to as Modified AC-WC, Modified AC-BC, and Modified AC-Base. While the Thin Asphalt Concrete layer, hereinafter referred to as HRS, consists of two types of mixtures, HRS Foundation (HRS-Base) and HRS Wearing Course (HRS-WC) and the maximum aggregate size of each mixture is $19 \mathrm{~mm}$ HRS-Base has an extent of coarse total portion more noteworthy than HRS-WC.

\section{LITERATURE REVIEW}

\subsection{Asphalt Concrete-Wearing Course}

Asphalt Concrete-Wearing Course (AC-WC) is the layer that is at the top that is directly related to the vehicle tires, has a smoother layer size than the Asphalt Concrete Binder Course (AC-BC). When receiving traffic loads above it and spread, the layer underneath is in the form of loads (vertical force, horizontal force) and vibration loads from the wheels. According to Silvia (2012) [6]

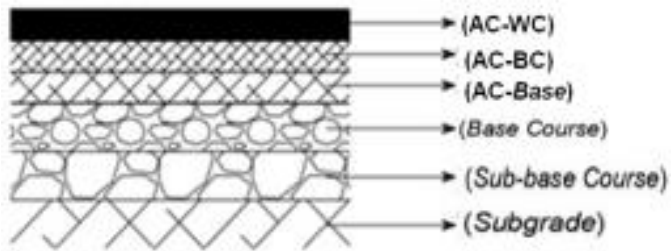

Figure 1. Asphalt Concrete (AC) Structure

\subsection{Hot Rolled Sheet-Wearing Course}

Hot Rolled Sheet-Wearing Course is a thin layer of asphalt concrete for road surfaces as a wear layer and as a cover, which is flexible so that it can accept the movement of the layer underneath without experiencing cracks. As a function of pavement construction, the asphalt used must be of the type that is heat resistant because it is located at the top position so that it is not easy to bleed (easily softens) and bulging (changes shape), does not easily experience cracks causing potholes, and it is not easy to lose the stickiness which causes the granulation to occur [3]

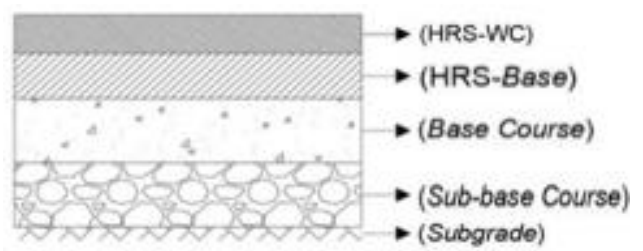

Figure 2. Hot Rolled Sheet (HRS) Structure

\subsection{Styrene-Butadiene-Styrene (SBS)}

Styrene-Butadiene-Styrene (SBS) modified asphalt is an elastomeric polymer type modified asphalt, which has high elastic response characteristics so that it is resistant to deformation caused by tension and will return to its original shape if the load is significant. This type of polymer is starting to get more attention in asphalt modification because it combines elastic and thermoplastic properties in its material properties, so it is called Thermoplastic Rubbers (TR). This property is possible from the types of forming monomers, namely Styrene and Butadine (Pradani, 2011) [5].

The reason for using SBS polymer for asphalt modification is because modified asphalt with SBS polymer has significantly increased properties, among others:

1. Resistant to high temperatures, because polymer asphalt has a softening point higher than $50^{\circ} \mathrm{C}$, so it can hold the asphalt from melting.

2. Can be used for traffic conditions that are quite dense so as to reduce high temperature deformation, 
because polymer asphalt has a higher softening point and modulus stiffness than ordinary asphalt.

3. Resistance to shear forces because polymer asphalt will increase resistance to shear forces.

4. Can increase the service life because the higher the thickness of the asphalt, the thicker the layer.

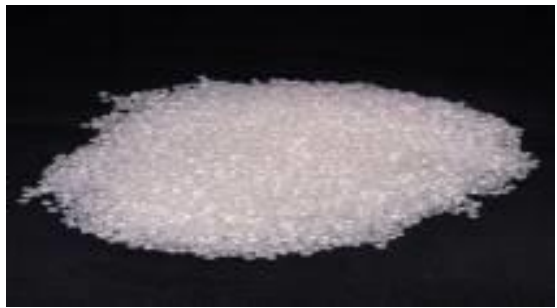

Figure 3. Styrene-Butadiene-Styrene (SBS)

\subsection{Ethylene-Vinyl-Acetate (EVA)}

Ethylene-Vinyl-Acetate (EVA) is a type of Thermoplastic Crystalline polymer or Plastomer composed of copolymers of ethylene and vinyl acetate. Weight percent vinyl acetic acid derivation generally shifts from 10-40\% with the remainder being ethylene. EVA is a polymer that is able to overcome the problems of high temperature, high traffic volume and overload on vehicles. This polymer has a good ability to unite with asphalt, so it is expected to improve the weaknesses of Pen 60/70 asphalt (Whiteoak, 1991) [7]

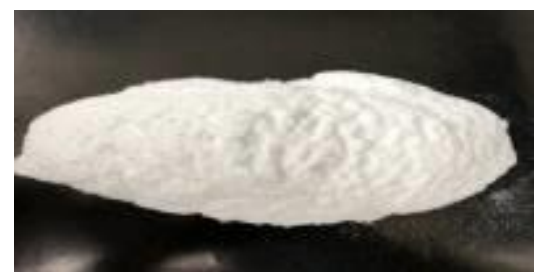

Figure 4. Ethylene-Vinyl-Acetate (EVA)

\subsection{Marshall and Marshall Immersion Test}

Marshall Test aims to measure the durability (stability) of the aggregate and asphalt mixture against flow. Flow is the change in deformation or strain of a mixture from no load to maximum load. Marshall Equipment is equipped with a proving ring with a capacity of $2500 \mathrm{~kg}(5000 \mathrm{lbs})$. In the proving ring there is a measuring watch and flowmeter where each function is used to measure the value of stability, and to measure plastic melt or flow. The standard Marshall specimen is a cylinder with a diameter of $10 \mathrm{~cm}$ and a height of $7.5 \mathrm{~cm}$.

Marshall Immersion test aims to determine changes in the characteristics of the mixture due to changes in water, temperature, and weather. In principle, the
Marshall Immersion test is the same as the Marshall test, which distinguishes only the immersion time. According to AASHTO 165-74 or ASTM D.1075-54 (1969) there are two Marshall Immersion test methods, namely the immersion test for $4 \times 24$ hours with a temperature of $\pm 50^{\circ} \mathrm{C}$ and the immersion test for $1 \times 24$ hours with a temperature of $\pm 60^{\circ} \mathrm{C}$.
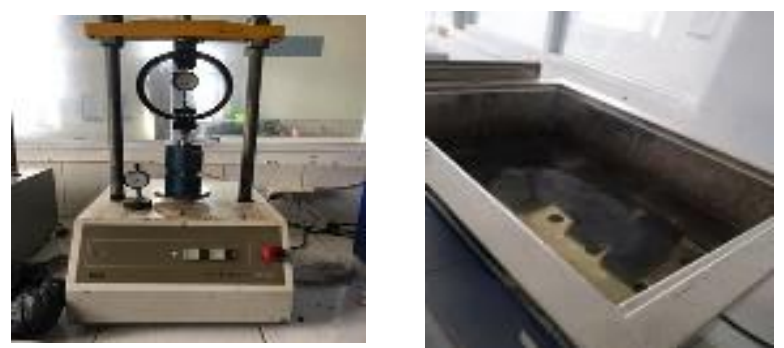

Figure 5. Marshall Testing Equipment and Sample Test Object

\subsection{Cantabro Test}

The Cantabro test is one of the tests in the laboratory to determine the magnitude of the limit for breaking or wear strength due to the effect of the impact (collision/loading) on the load of traffic wheels on the pavement layer. Traffic loading on the pavement surface repeatedly will cause the pavement layer to wear out, this will cause the pavement layer to decrease the durability of the asphalt, so an abrasion test is performed with a Los Angeles machine without using steel balls.

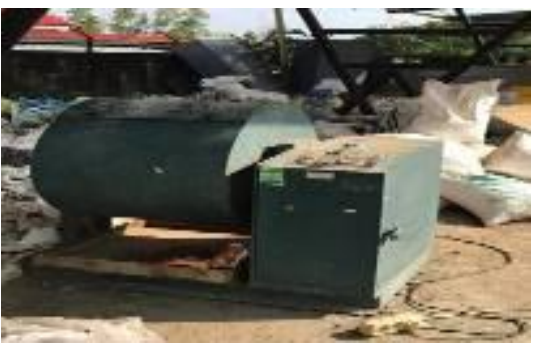

Figure 6. Cantabro Testing with Los Angeles Machine

\section{RESULTS AND DISCUSSION}

\subsection{Test Results of Asphalt Modifications}

\section{Characteristics}

SBS modified asphalt testing and EVA modified asphalt testing were carried out by using several percent variations of the addition of SBS polymer and EVA polymer. The additions of EVA polymer and SBS polymer are $0.5 \%, 1 \%$ and $2 \%$ to the asphalt mixture. 
The following table shows the results of testing the characteristics of the modified asphalt mixture.

Table 1. Test Results of Asphalt Pen. 60/70 Characteristics with addition SBS 0.5\%, $1 \%$ and 2\%.

\begin{tabular}{|c|c|c|c|c|c|c|c|c|}
\hline \multirow[b]{2}{*}{ No } & \multirow[b]{2}{*}{ Test } & \multirow[b]{2}{*}{$\begin{array}{c}\text { Test } \\
\text { Method }\end{array}$} & \multirow{2}{*}{$\begin{array}{c}\text { Asphalt Pen } \\
60 / 70 \\
\text { Requirements }\end{array}$} & \multirow[b]{2}{*}{$\begin{array}{l}0 \% \\
\text { SBS }\end{array}$} & \multirow{2}{*}{$\begin{array}{c}\text { Modified } \\
\text { Asphalt } \\
\text { Requirements }\end{array}$} & \multicolumn{3}{|c|}{ SBS Content } \\
\hline & & & & & & $0.5 \%$ & $1 \%$ & $2 \%$ \\
\hline 1. & $\begin{array}{l}\text { Penetration } 25^{\circ} \mathrm{C}, \\
100 \mathrm{gr}, 5 \text { second, } \\
0,1 \mathrm{~mm}\end{array}$ & $\begin{array}{c}\text { SNI } \\
2456: 2011\end{array}$ & $60-70$ & 62 & Reported & 59 & 57 & 50 \\
\hline 2. & $\begin{array}{l}\text { Ductility at } 25^{\circ} \mathrm{C} \\
(\mathrm{cm})\end{array}$ & $\begin{array}{c}\text { SNI } \\
2432: 2011\end{array}$ & $\geq 100$ & 140 & - & 140 & 140 & 103 \\
\hline 3. & Flash Point $\left({ }^{\circ} \mathrm{C}\right)$ & $\begin{array}{c}\text { SNI } \\
2433: 2011\end{array}$ & $\geq 232$ & 323 & $\geq 230$ & 340 & 332 & 326 \\
\hline 4. & Burn Point $\left({ }^{\circ} \mathrm{C}\right)$ & $\begin{array}{c}\text { SNI } \\
2433: 2011\end{array}$ & - & 330 & - & 346 & 336 & 331 \\
\hline 5. & Softening Point $\left({ }^{\circ} \mathrm{C}\right)$ & $\begin{array}{c}\text { SNI } \\
2434: 2011\end{array}$ & $\geq 48$ & 51 & Reported & 51,5 & 51,6 & 52,6 \\
\hline 6. & Density & $\begin{array}{c}\text { SNI } \\
2441: 2011\end{array}$ & $\geq 1.0$ & 1.032 & - & 1.033 & 1.034 & 1.035 \\
\hline
\end{tabular}

Table 2. Test Results of Asphalt Pen. 60/70 Characteristics with addition EVA $0.5 \%, 1 \%$ and $2 \%$.

\begin{tabular}{|c|c|c|c|c|c|c|c|c|}
\hline \multirow[b]{2}{*}{ No } & \multirow[b]{2}{*}{ Test } & \multirow[b]{2}{*}{$\begin{array}{c}\text { Test } \\
\text { Method }\end{array}$} & \multirow{2}{*}{$\begin{array}{c}\text { Asphalt Pen } \\
60 / 70 \\
\text { Requirements }\end{array}$} & \multirow[b]{2}{*}{$\begin{array}{c}0 \% \\
\text { EVA }\end{array}$} & \multirow{2}{*}{$\begin{array}{c}\text { Modified } \\
\text { Asphalt } \\
\text { Requirements }\end{array}$} & \multicolumn{3}{|c|}{ EVA Content } \\
\hline & & & & & & $0.5 \%$ & $1 \%$ & $2 \%$ \\
\hline 1. & $\begin{array}{l}\text { Penetration } \\
25^{\circ} \mathrm{C}, 100 \mathrm{gr}, 5 \\
\text { second, } 0,1 \mathrm{~mm}\end{array}$ & $\begin{array}{c}\text { SNI } \\
2456: 2011\end{array}$ & $60-70$ & 62 & Reported & 60 & 58 & 51 \\
\hline 2. & $\begin{array}{l}\text { Ductility at } 25^{\circ} \mathrm{C} \\
(\mathrm{cm})\end{array}$ & $\begin{array}{c}\text { SNI } \\
2432: 2011\end{array}$ & $\geq 100$ & 140 & - & 140 & 140 & 110 \\
\hline 3. & Flash Point $\left({ }^{\circ} \mathrm{C}\right)$ & $\begin{array}{c}\text { SNI } \\
2433: 2011\end{array}$ & $\geq 232$ & 323 & $\geq 230$ & 345 & 334 & 325 \\
\hline 4. & Burn Point $\left({ }^{\circ} \mathrm{C}\right)$ & $\begin{array}{c}\text { SNI } \\
2433: 2011\end{array}$ & - & 330 & - & 352 & 343 & 333 \\
\hline 5. & $\begin{array}{l}\text { Softening Point } \\
\left({ }^{\circ} \mathrm{C}\right)\end{array}$ & $\begin{array}{c}\text { SNI } \\
2434: 2011\end{array}$ & $\geq 48$ & 51 & Reported & 51,4 & 51,6 & 51,9 \\
\hline 6. & Density & $\begin{array}{c}\text { SNI } \\
2441: 2011\end{array}$ & $\geq 1.0$ & 1.032 & - & 1.032 & 1.034 & 1.036 \\
\hline
\end{tabular}

Based on the results of testing the modified asphalt mixture, it can be concluded that the asphalt miture with the addition of $0.5 \%$ SBS polymer and EVA polymer has the best performance according to the General Specifications of Bina Marga Division 6, Revision 1 of 2018.

\subsection{Marshall Test Results}

Marshall Test was carried out to obtain optimum asphalt content, optimum asphalt content was obtained from Marshall characteristics, namely Stability, Flow, Void in Mineral Aggregate (VMA), 
Void in Mix (VIM), Void Filled with Asphalt (VFA), and Marshall Quotient (MQ) which is eligible for the AC mix, HRS and AC mix, modified HRS in accordance with the General Specifications of Bina Marga Division 6 Revision 1 of 2018 .

Table 3. Marshall Standard Asphalt Concrete-Wearing Course (AC-WC)

\begin{tabular}{|c|c|c|c|c|c|c|c|}
\hline \multirow{2}{*}{ No } & \multirow{2}{*}{ Characteristics } & \multirow{2}{*}{ Requirements } & \multicolumn{5}{|c|}{ Asphalt Content } \\
\hline & & & $5 \%$ & $5.5 \%$ & $6 \%$ & $6.5 \%$ & $7 \%$ \\
\hline 1 & \multirow{3}{*}{ VMA } & \multirow{3}{*}{ Min. 15} & 15.879 & 15.844 & 15.907 & 16.386 & 16.794 \\
\hline 2 & & & 15.734 & 15.881 & 16.026 & 16.374 & 16.748 \\
\hline 3 & & & 15.822 & 15.872 & 16.063 & 16.470 & 16.659 \\
\hline & \multicolumn{2}{|l|}{ Average } & 15.81 & 15.87 & 16.00 & 16.41 & 16.73 \\
\hline 1 & \multirow{3}{*}{ VFA } & \multirow{3}{*}{ Min. 65} & 70.382 & 78.031 & 85.175 & 89.542 & 94.132 \\
\hline 2 & & & 71.153 & 77.814 & 84.426 & 89.622 & 94.446 \\
\hline 3 & & & 70.681 & 77.870 & 84.193 & 89.000 & 95.048 \\
\hline & \multicolumn{2}{|l|}{ Average } & 70.74 & 77.90 & 84.60 & 89.39 & 94.54 \\
\hline 1 & \multirow{3}{*}{ VIM } & & 6.396 & 5.175 & 4.051 & 3.396 & 2.660 \\
\hline 2 & & $3-5$ & 6.235 & 5.216 & 4.186 & 3.382 & 2.606 \\
\hline \multirow[t]{2}{*}{3} & & & 6.333 & 5.206 & 4.228 & 3.493 & 2.502 \\
\hline & \multicolumn{2}{|l|}{ Average } & 6.32 & 5.20 & 4.15 & 3.42 & 2.59 \\
\hline 1 & \multirow{3}{*}{ Stability } & \multirow{3}{*}{ Min. 800} & 941.531 & 944.076 & 1002.788 & 975.563 & 955.144 \\
\hline 2 & & & 908.700 & 973.294 & 972.818 & 973.294 & 941.531 \\
\hline \multirow[t]{2}{*}{3} & & & 904.279 & 975.563 & 966.185 & 952.919 & 926.388 \\
\hline & \multicolumn{2}{|l|}{ Average } & 918.17 & 964.31 & 980.60 & 967.26 & 941.02 \\
\hline 1 & \multirow{3}{*}{ Flow } & Min. 2 & 3.10 & 3.25 & 3.30 & 3.43 & 3.58 \\
\hline 2 & & & 3.18 & 3.19 & 3.32 & 3.39 & 3.6 \\
\hline \multirow[t]{2}{*}{3} & & $\operatorname{Max} 4$ & 3.09 & 3.17 & 3.28 & 3.47 & 3.61 \\
\hline & \multicolumn{2}{|l|}{ Average } & 3.12 & 3.20 & 3.30 & 3.43 & 3.60 \\
\hline 1 & \multirow{3}{*}{ MQ } & & 303.720 & 290.485 & 303.875 & 284.421 & 266.800 \\
\hline 2 & & Min. 250 & 285.755 & 305.108 & 293.017 & 287.107 & 261.536 \\
\hline 3 & & & 292.647 & 307.748 & 294.569 & 274.617 & 256.617 \\
\hline & \multicolumn{2}{|l|}{ Average } & 294.04 & 301.11 & 297.15 & 282.05 & 261.65 \\
\hline
\end{tabular}

Based on Table 3, the increasing of asphalt content, the overall estimation value of VMA, VFA, and Flow will also increase, while the VIM value decreases, and for the asphalt content value of $6 \%$ produces the greatest stability and MQ values.

Table 4. Marshall Standard Hot Rolled Sheet-Wearing Course (HRS-WC)

\begin{tabular}{|c|c|c|c|c|c|c|c|}
\hline \multirow{2}{*}{ No } & \multirow{2}{*}{ Characteristics } & \multirow{2}{*}{ Requirements } & \multicolumn{5}{|c|}{ Asphalt Content } \\
\hline & & & $7 \%$ & $7.5 \%$ & $8 \%$ & $8.5 \%$ & $9 \%$ \\
\hline 1 & \multirow{3}{*}{ VMA } & \multirow{3}{*}{ Min. 18} & 19.987 & 20.632 & 21.122 & 21.709 & 21.730 \\
\hline 2 & & & 20.095 & 20.629 & 21.081 & 21.643 & 21.749 \\
\hline 3 & & & 20.410 & 20.644 & 21.059 & 21.548 & 21.795 \\
\hline & \multicolumn{2}{|l|}{ Average } & 20.16 & 20.63 & 21.09 & 21.63 & 21.76 \\
\hline 1 & \multirow{3}{*}{ VFA } & \multirow{3}{*}{ Min 68} & 76.204 & 78.885 & 82.125 & 84.730 & 90.096 \\
\hline 2 & & & 75.693 & 78.898 & 82.329 & 85.058 & 89.994 \\
\hline 3 & & & 74.231 & 78.824 & 82.436 & 85.536 & 89.749 \\
\hline & \multicolumn{2}{|l|}{ Average } & 75.38 & 78.87 & 82.30 & 85.11 & 89.95 \\
\hline 1 & \multirow{3}{*}{ VIM } & \multirow{3}{*}{$4-6$} & 6.231 & 5.819 & 5.229 & 4.758 & 3.594 \\
\hline 2 & & & 6.357 & 5.816 & 5.180 & 4.678 & 3.618 \\
\hline 3 & & & 6.726 & 5.834 & 5.154 & 4.563 & 3.675 \\
\hline & \multicolumn{2}{|l|}{ Average } & 6.44 & 5.82 & 5.19 & 4.67 & 3.63 \\
\hline 1 & \multirow{3}{*}{ Stability } & \multirow{3}{*}{ Min. 600} & 738.453 & 876.445 & 934.875 & 859.856 & 688.172 \\
\hline 2 & & & 727.398 & 885.102 & 947.859 & 860.055 & 688.172 \\
\hline 3 & & & 753.930 & 888.797 & 954.352 & 864.477 & 703.320 \\
\hline & \multicolumn{2}{|l|}{ Average } & 739.93 & 883.45 & 945.70 & 861.46 & 693.22 \\
\hline 1 & \multirow{3}{*}{ Flow } & \multirow{3}{*}{ - } & 3.20 & 3.3 & 3.60 & 3.5 & 3.4 \\
\hline 2 & & & 3.00 & 3.4 & 3.50 & 3.6 & 3.5 \\
\hline 3 & & & 3.10 & 3.3 & 3.50 & 3.6 & 3.5 \\
\hline & \multicolumn{2}{|l|}{ Average } & 3.10 & 3.33 & 3.53 & 3.57 & 3.47 \\
\hline 1 & \multirow{3}{*}{ MQ } & \multirow{3}{*}{$\operatorname{Min} 250$} & 230.767 & 265.589 & 259.688 & 245.673 & 202.403 \\
\hline 2 & & & 242.466 & 260.324 & 270.817 & 238.904 & 196.621 \\
\hline 3 & & & 243.203 & 269.332 & 272.672 & 240.132 & 200.949 \\
\hline & \multicolumn{2}{|l|}{ Average } & 238.81 & 265.08 & 267.73 & 241.57 & 199.99 \\
\hline
\end{tabular}

Based on Table 4, the increasing of asphalt content, the overall estimation value of VMA, VFA, and Flow will also increase, while the VIM value decreases, and for the asphalt content value of $8 \%$ produces the greatest stability and MQ values. 
Table 5. Marshall Asphalt Modification 0.5\% SBS Asphalt Concrete-Wearing Course (AC-WC)

\begin{tabular}{|c|c|c|c|c|c|c|c|}
\hline \multirow{2}{*}{ No } & \multirow{2}{*}{ Characteristics } & \multirow{2}{*}{ Requirements } & \multicolumn{5}{|c|}{ Asphalt Content } \\
\hline & & & $5 \%$ & $5.5 \%$ & $6 \%$ & $6.5 \%$ & $7 \%$ \\
\hline 1 & \multirow{3}{*}{ VMA } & \multirow{3}{*}{ Min. 15} & 15.098 & 15.547 & 15.959 & 16.372 & 16.763 \\
\hline 2 & & & 15.073 & 15.505 & 15.996 & 16.271 & 16.871 \\
\hline 3 & & & 15.120 & 15.569 & 16.108 & 16.278 & 16.821 \\
\hline & \multicolumn{2}{|l|}{ Average } & 15.10 & 15.54 & 16.02 & 16.31 & 16.82 \\
\hline 1 & \multirow{3}{*}{ VFA } & \multirow{3}{*}{ Min. 65} & 74.639 & 79.729 & 84.763 & 89.549 & 94.254 \\
\hline 2 & & & 74.785 & 79.986 & 84.529 & 90.216 & 93.529 \\
\hline 3 & & & 74.510 & 79.592 & 83.834 & 90.167 & 93.858 \\
\hline & \multicolumn{2}{|l|}{ Average } & 74.64 & 79.77 & 84.38 & 89.98 & 93.88 \\
\hline 1 & \multirow{3}{*}{ VIM } & & 5.538 & 4.851 & 4.123 & 3.394 & 2.638 \\
\hline 2 & & $3-5$ & 5.510 & 4.804 & 4.165 & 3.277 & 2.765 \\
\hline 3 & & & 5.562 & 4.877 & 4.292 & 3.286 & 2.707 \\
\hline & \multicolumn{2}{|l|}{ Average } & 5.54 & 4.84 & 4.19 & 3.32 & 2.70 \\
\hline 1 & \multirow{3}{*}{ Stability } & \multirow{3}{*}{ Min. 1000} & 1073.119 & 1138.639 & 1195.631 & 1177.481 & 1161.600 \\
\hline 2 & & & 1063.467 & 1152.525 & 1178.436 & 1186.556 & 1163.869 \\
\hline \multirow[t]{2}{*}{3} & & & 1054.623 & 1168.406 & 1191.702 & 1171.804 & 1147.483 \\
\hline & \multicolumn{2}{|l|}{ Average } & 1063.74 & 1153.19 & 1188.59 & 1178.61 & 1157.65 \\
\hline 1 & \multirow{3}{*}{ Flow } & Min. 2 & 2.74 & 2.93 & 3.05 & 3.24 & 3.63 \\
\hline 2 & & & 2.68 & 2.88 & 3.02 & 3.25 & 3.5 \\
\hline 3 & & $\operatorname{Max} 4$ & 2.63 & 2.91 & 3.03 & 3.3 & 3.54 \\
\hline & \multicolumn{2}{|l|}{ Average } & 2.68 & 2.91 & 3.03 & 3.26 & 3.56 \\
\hline 1 & \multirow{3}{*}{ MQ } & & 391.649 & 388.614 & 392.010 & 363.420 & 320.000 \\
\hline 2 & & Min. 250 & 396.816 & 400.182 & 390.211 & 365.094 & 332.534 \\
\hline 3 & & & 400.997 & 401.514 & 393.301 & 355.092 & 324.148 \\
\hline & \multicolumn{2}{|l|}{ Average } & 396.49 & 396.77 & 391.84 & 361.20 & 325.56 \\
\hline
\end{tabular}

Based on Table 5, the increasing of asphalt content, the overall estimation or value of VMA, VFA, and Flow will also increase, while the VIM value decreases, and for the asphalt content value of $6 \%$ produces the greatest stability and MQ values.
The difference in requirements between standard asphalt Marshall and modified asphalt Marshall for $\mathrm{AC}$ is only in the stability value, which is a minimum of $1000 \mathrm{Kg}$.

Table 6. Marshall Asphalt Modification 0.5\% SBS Hot Rolled Sheet-Wearing Course (HRS-WC)

\begin{tabular}{|c|c|c|c|c|c|c|c|}
\hline \multirow{2}{*}{ No } & \multirow{2}{*}{ Characteristics } & \multirow{2}{*}{ Requirements } & \multicolumn{5}{|c|}{ Asphalt Content } \\
\hline & & & $7 \%$ & $7.5 \%$ & $8 \%$ & $8.5 \%$ & $9 \%$ \\
\hline 1 & \multirow{3}{*}{ VMA } & \multirow{3}{*}{ Min. 18} & 19.724 & 20.586 & 21.148 & 21.900 & 22.265 \\
\hline 2 & & & 19.660 & 20.550 & 21.127 & 21.748 & 22.262 \\
\hline 3 & & & 19.627 & 20.489 & 21.208 & 21.832 & 22.213 \\
\hline & \multicolumn{2}{|l|}{ Average } & 19.67 & 20.54 & 21.16 & 21.83 & 22.25 \\
\hline 1 & \multirow{3}{*}{ VFA } & \multirow{3}{*}{ Min 68} & 77.474 & 79.105 & 81.998 & 83.787 & 87.328 \\
\hline 2 & & & 77.788 & 79.279 & 82.103 & 84.534 & 87.342 \\
\hline 3 & & & 77.954 & 79.576 & 81.704 & 84.121 & 87.590 \\
\hline & \multicolumn{2}{|l|}{ Average } & 77.74 & 79.32 & 81.93 & 84.15 & 87.42 \\
\hline 1 & \multirow{3}{*}{ VIM } & \multirow{3}{*}{$4-6$} & 5.923 & 5.765 & 5.260 & 4.990 & 4.254 \\
\hline 2 & & & 5.848 & 5.722 & 5.235 & 4.806 & 4.251 \\
\hline 3 & & & 5.808 & 5.650 & 5.332 & 4.907 & 4.190 \\
\hline & \multicolumn{2}{|l|}{ Average } & 5.86 & 5.71 & 5.28 & 4.90 & 4.23 \\
\hline 1 & \multirow{3}{*}{ Stability } & \multirow{3}{*}{ Min. 600} & 806.992 & 928.383 & 1012.781 & 912.038 & 772.570 \\
\hline 2 & & & 784.883 & 921.891 & 1006.289 & 897.641 & 757.422 \\
\hline 3 & & & 756.141 & 952.914 & 1001.961 & 886.586 & 733.617 \\
\hline & \multicolumn{2}{|l|}{ Average } & 782.67 & 934.40 & 1007.01 & 898.75 & 754.54 \\
\hline 1 & \multirow{3}{*}{ Flow } & \multirow{3}{*}{-} & 3.10 & 3.2 & 3.40 & 3.4 & 3.4 \\
\hline 2 & & & 3.00 & 3.2 & 3.30 & 3.3 & 3.4 \\
\hline 3 & & & 3.00 & 3.3 & 3.30 & 3.3 & 3.3 \\
\hline & \multicolumn{2}{|l|}{ Average } & 3.03 & 3.23 & 3.33 & 3.33 & 3.37 \\
\hline 1 & \multirow{3}{*}{ MQ } & \multirow{3}{*}{$\operatorname{Min} 250$} & 260.320 & 290.120 & 297.877 & 268.246 & 227.227 \\
\hline 2 & & & 261.628 & 288.091 & 304.936 & 272.012 & 222.771 \\
\hline 3 & & & 252.047 & 288.762 & 303.625 & 268.662 & 222.308 \\
\hline & \multicolumn{2}{|l|}{ Average } & 258.00 & 288.99 & 302.15 & 269.64 & 224.10 \\
\hline
\end{tabular}

Based on Table 6, the increasing of asphalt content, the overall value of VMA, VFA, and Flow will also increase, while the VIM value decreases, and for the asphalt content value of $8 \%$ produces the greatest value of stability and MQ. 
Table 7. Marshall Asphalt Modification 0.5\% EVA Asphalt Concrete-Wearing Course (AC-WC)

\begin{tabular}{|c|c|c|c|c|c|c|c|}
\hline \multirow{2}{*}{ No } & \multirow{2}{*}{ Characteristics } & \multirow{2}{*}{ Requirements } & \multicolumn{5}{|c|}{ Asphalt Content } \\
\hline & & & $5 \%$ & $5.5 \%$ & $6 \%$ & $6.5 \%$ & $7 \%$ \\
\hline 1 & \multirow{3}{*}{ VMA } & \multirow{3}{*}{ Min. 15} & 14.960 & 15.532 & 15.999 & 16.285 & 16.467 \\
\hline 2 & & & 15.000 & 15.591 & 16.016 & 16.347 & 16.485 \\
\hline 3 & & & 15.123 & 15.519 & 15.974 & 16.300 & 16.420 \\
\hline & \multicolumn{2}{|l|}{ Average } & 15.03 & 15.55 & 16.00 & 16.31 & 16.46 \\
\hline 1 & \multirow{3}{*}{ VFA } & \multirow{3}{*}{ Min. 65} & 75.521 & 79.897 & 84.595 & 90.206 & 96.378 \\
\hline 2 & & & 75.284 & 79.536 & 84.486 & 89.799 & 96.256 \\
\hline 3 & & & 74.566 & 79.973 & 84.753 & 90.108 & 96.707 \\
\hline & \multicolumn{2}{|l|}{ Average } & 75.12 & 79.80 & 84.61 & 90.04 & 96.45 \\
\hline 1 & \multirow{3}{*}{ VIM } & & 5.373 & 4.822 & 4.155 & 3.280 & 2.278 \\
\hline 2 & & $3-5$ & 5.418 & 4.889 & 4.175 & 3.351 & 2.298 \\
\hline 3 & & & 5.555 & 4.808 & 4.127 & 3.297 & 2.223 \\
\hline & \multicolumn{2}{|l|}{ Average } & 5.45 & 4.84 & 4.15 & 3.31 & 2.27 \\
\hline 1 & \multirow{3}{*}{ Stability } & \multirow{3}{*}{ Min. 1000} & 1043.625 & 1120.952 & 1166.138 & 1127.569 & 1020.938 \\
\hline 2 & & & 1030.303 & 1145.719 & 1147.483 & 1134.375 & 1027.744 \\
\hline \multirow[t]{2}{*}{3} & & & 1039.147 & 1159.331 & 1149.694 & 1125.374 & 1023.670 \\
\hline & \multicolumn{2}{|l|}{ Average } & 1037.69 & 1142.00 & 1154.44 & 1129.11 & 1024.12 \\
\hline 1 & \multirow{3}{*}{ Flow } & Min. 2 & 2.75 & 3.13 & 3.21 & 3.35 & 3.61 \\
\hline 2 & & & 2.73 & 3.07 & 3.23 & 3.31 & 3.63 \\
\hline 3 & & $\operatorname{Max} 4$ & 2.79 & 3.05 & 3.19 & 3.38 & 3.62 \\
\hline & \multicolumn{2}{|l|}{ Average } & 2.76 & 3.08 & 3.21 & 3.35 & 3.62 \\
\hline 1 & \multirow{3}{*}{ MQ } & & 379.500 & 358.132 & 363.283 & 336.588 & 282.808 \\
\hline 2 & & Min. 250 & 377.400 & 373.198 & 355.258 & 342.711 & 283.125 \\
\hline 3 & & & 372.454 & 380.109 & 360.406 & 332.951 & 282.782 \\
\hline & \multicolumn{2}{|l|}{ Average } & 376.45 & 370.48 & 359.65 & 337.42 & 282.90 \\
\hline
\end{tabular}

Based on Table 7, the increasing of asphalt content, the overall value of VMA, VFA, and Flow will also increase, while the VIM value decreases, and for the asphalt content value of $6 \%$ produces the greatest stability and MQ values. The difference in requirements between standard asphalt Marshall and modified asphalt Marshall for AC is only in the stability value, which is a minimum of $1000 \mathrm{Kg}$.

Table 8. Marshall Asphalt Modification 0.5\% EVA Hot Rolled Sheet-Wearing Course (HRS-WC)

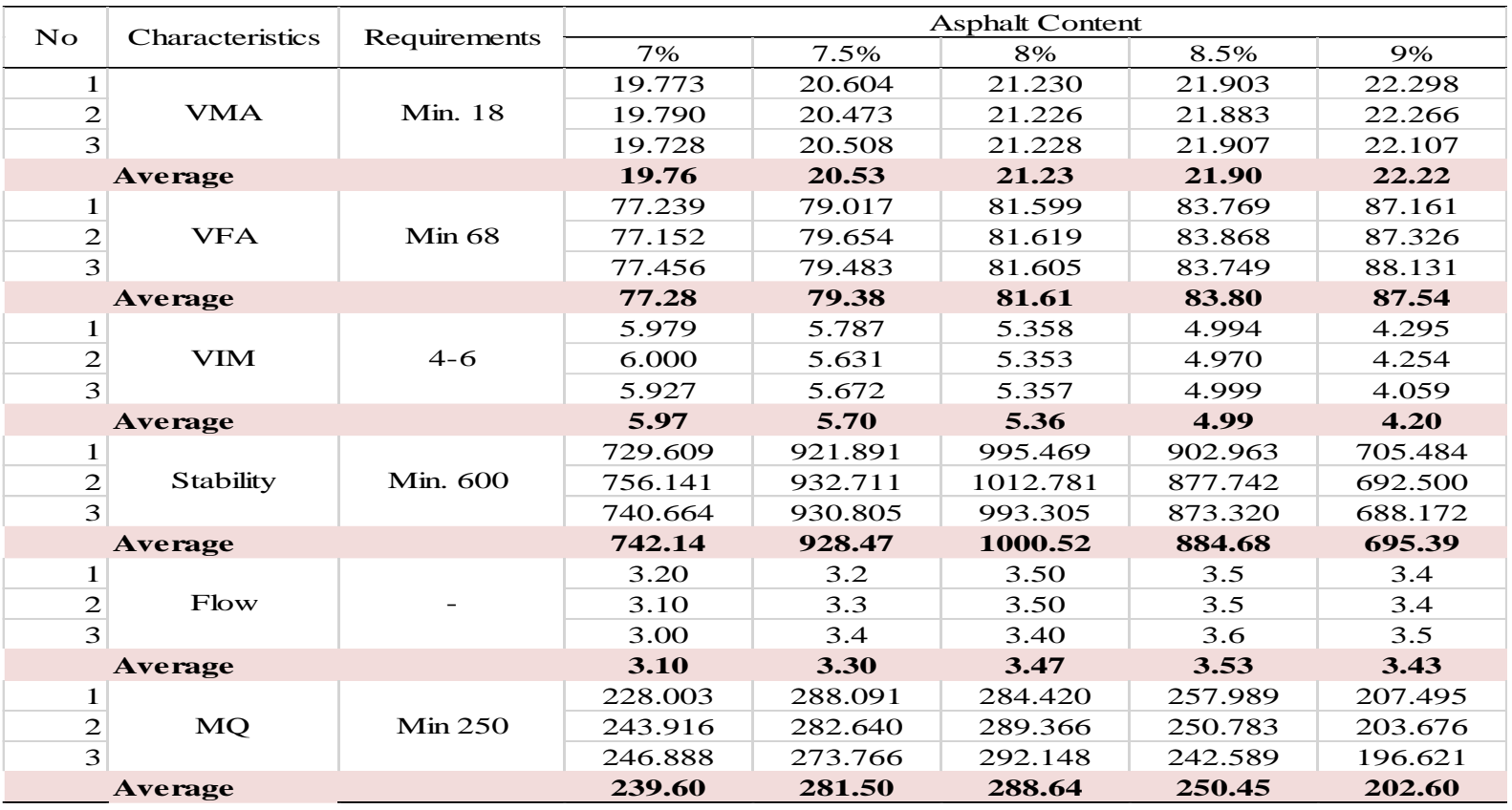

Based on Table 8, the increasing of asphalt content, the overall value of VMA, VFA, and Flow will also increase, while the VIM value decreases, and for the asphalt content value of $8 \%$ produces the greatest stability and MQ values. 


\subsection{Optimum Asphalt Content}

From the results of standard asphalt Marshall testing and modified asphalt Marshall testing in Table 9. Optimum Asphalt Content
Table 3 through 8 can be determined the value of optimum asphalt content, the optimum asphalt content value can be seen in Table 9.

\begin{tabular}{|c|c|c|c|}
\hline & Standard Marshall & $\begin{array}{c}\text { Marshall Asphalt } \\
\text { Modification } 0,5 \% \\
\text { SBS } \\
\end{array}$ & $\begin{array}{c}\text { Marshall Asphalt } \\
\text { Modification } 0,5 \% \text { EVA }\end{array}$ \\
\hline $\begin{array}{c}\text { Asphalt Concrete- } \\
\text { Wearing Course } \\
\text { (AC-WC) }\end{array}$ & $6.16 \%$ & $6.08 \%$ & $6.02 \%$ \\
\hline $\begin{array}{l}\text { Hot Rolled Sheet- } \\
\text { Wearing Course } \\
\text { (HRS-WC) }\end{array}$ & $7.9 \%$ & $7.88 \%$ & $7.835 \%$ \\
\hline
\end{tabular}

After obtaining the optimum asphalt content, the optimum asphalt content is used as a reference for obtaining the Marshall Parameter value for the three mixtures of Asphalt Concrete-Wearing Course (AC-WC) and Hot Rolled Sheet-Wearing Course (HRS-WC).

Table 10. Marshall Test Results against Optimum Asphalt Content (KAO)

\begin{tabular}{clcccccc}
\hline & & \multicolumn{2}{c}{$\begin{array}{c}\text { Asphalt Concrete-Wearing Course } \\
\text { (AC-WC) Mixture }\end{array}$} & \multicolumn{2}{c}{$\begin{array}{c}\text { Hot Rolled Sheet-Wearing Course } \\
\text { (HRS-WC) Mixture }\end{array}$} \\
\cline { 3 - 8 } NO & Parameter & $\begin{array}{c}\text { Standard } \\
\text { Asphalt }\end{array}$ & $\begin{array}{c}\text { Asphalt } \\
\text { Modification } \\
\text { 0,5\% EVA }\end{array}$ & $\begin{array}{c}\text { Asphalt } \\
\text { Modification } \\
\text { 0,5\% SBS }\end{array}$ & $\begin{array}{c}\text { Standard } \\
\text { Asphalt }\end{array}$ & $\begin{array}{c}\text { Asphalt } \\
\text { Modification } \\
\text { 0,5\% EVA }\end{array}$ & $\begin{array}{c}\text { Msphalt } \\
\text { Modification } \\
\mathbf{0 , 5 \%} \text { SBS }\end{array}$ \\
\hline 1 & KAO & 6.16 & 6.02 & 6.08 & 7.9 & 7.835 & 7.88 \\
2 & VMA & 16.128 & 16.009 & 16.033 & 21.054 & 21.045 & 21.052 \\
3 & VFA & 86.130 & 84.833 & 85.513 & 81.324 & 80.656 & 81.120 \\
4 & VIM & 3.923 & 4.118 & 4.013 & 5.385 & 5.524 & 5.429 \\
5 & Stability & 979.484 & 1162.648 & 1189.285 & 934.310 & 981.339 & 985.073 \\
6 & Flow & 3.338 & 3.226 & 3.087 & 3.507 & 3.432 & 3.302 \\
7 & MQ & 293.451 & 359.947 & 385.289 & 267.016 & 286.101 & 298.242 \\
\hline
\end{tabular}

\subsection{Marshall Immersion Test Results}

Marshall Immersion test was conducted to obtain index of retained strength based on the optimum asphalt content. The requirement for the index of retained strength according to the General Bina Marga Division 6 Revision 1 Year 2018 specifications is more than $90 \%$.

Table 11. Index of Retained Strength on Asphalt Concrete-Wearing Course (AC-WC)

\begin{tabular}{|c|c|c|c|c|c|c|c|}
\hline \multirow{2}{*}{ No. } & \multirow{2}{*}{$\begin{array}{c}\text { Mixture } \\
\text { Type }\end{array}$} & \multirow{2}{*}{$\begin{array}{c}\text { KAO } \\
(\%)\end{array}$} & \multicolumn{2}{|c|}{ Stability (kg) } & \multirow{2}{*}{$\begin{array}{c}\text { Index of } \\
\text { retained } \\
\text { strength } \\
(\%)\end{array}$} & \multirow{2}{*}{ Requirements } & \multirow{2}{*}{ Information } \\
\hline & & & Standard & Immersion & & & \\
\hline 1 & $\begin{array}{c}\text { Standard } \\
\text { Asphalt } \\
\text { Mixture }\end{array}$ & 6.16 & 988.20 & 912.79 & 92.37 & $\geq 90 \%$ & Fulfill \\
\hline 2 & $\begin{array}{c}\text { SBS } \\
\text { modified } \\
\text { asphalt mix }\end{array}$ & 6.08 & 1195.25 & 1115.47 & 93.33 & & Fulfill \\
\hline
\end{tabular}


EVA

3 modified

asphalt mix

$6.02 \quad 1160.43$

1076.13

Based on Table 11, the test specimen for the mixture of SBS modified asphalt AC-WC produces the highest index of retained strength value, which is $93.33 \%$ compared to the test specimen for the mixture of standard asphalt AC at $92.37 \%$ and the mixture for EVA modified asphalt AC-WC at $92.74 \%$.

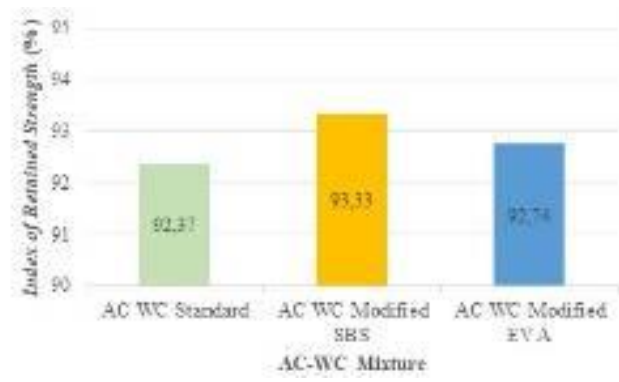

Figure 7. Comparison IRS on AC-WC mixture

Table 12. Index of Retained Strength on Hot Rolled Sheet-Wearing Course (HRS-WC)

\begin{tabular}{|c|c|c|c|c|c|c|c|}
\hline \multirow{2}{*}{ No. } & \multirow{2}{*}{$\begin{array}{l}\text { Mixture } \\
\text { Type }\end{array}$} & \multirow{2}{*}{$\begin{array}{c}\mathrm{KAO} \\
(\%)\end{array}$} & \multicolumn{2}{|c|}{ Stability (kg) } & \multirow{2}{*}{$\begin{array}{c}\text { Index of } \\
\text { Retained } \\
\text { Strength } \\
(\%)\end{array}$} & \multirow{2}{*}{ Requirements } & \multirow{2}{*}{ Information } \\
\hline & & & Standard & Immersion & & & \\
\hline 1 & $\begin{array}{l}\text { Standard } \\
\text { Asphalt } \\
\text { Mixture }\end{array}$ & 7.9 & 914.6 & 825.46 & 90.25 & & Fulfill \\
\hline 2 & $\begin{array}{c}\text { SBS } \\
\text { modified } \\
\text { asphalt mix }\end{array}$ & 7.88 & 997.40 & 912.38 & 91.48 & $\geq 90 \%$ & Fulfill \\
\hline 3 & $\begin{array}{c}\text { EVA } \\
\text { modified } \\
\text { asphalt mix }\end{array}$ & 7.83 & 989.77 & 896.55 & 90.58 & & Fulfill \\
\hline
\end{tabular}

Based on Table 12, the test specimen of SBS modified asphalt HRS-WC mixture produced the highest index of retained strength of $91.48 \%$ compared to the standard asphalt HRS-WC mixture test specimen of $90.25 \%$ and EVA modified asphalt HRS-WC of $90.58 \%$.

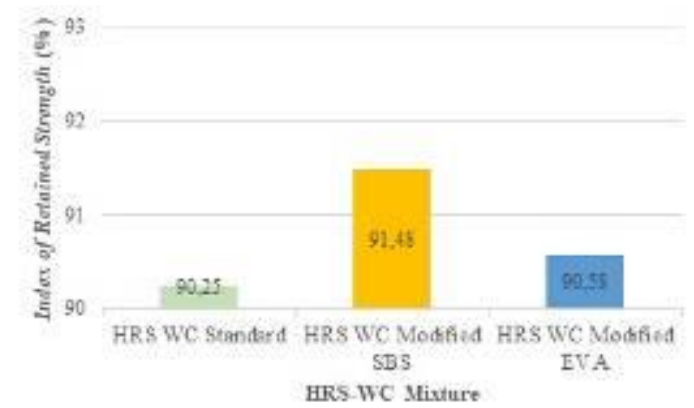

Figure 8 . Comparison IRS on HRS-WC mixture

\subsection{Cantabro Test Results}

Cantabro testing is carried out to obtain a percent of weight loss (wear value) based on the optimum asphalt content. Requirements for the wear index value of the 2010 General Bina Marga specification are less than $20 \%$. 
Table 13. Percent of Weight Loss of Asphalt Concrete-Wearing Course (AC-WC)

\begin{tabular}{|c|c|c|c|c|c|c|c|c|}
\hline No. & $\begin{array}{l}\text { Type of } \\
\text { Specimen }\end{array}$ & $\begin{array}{c}\text { Optimum } \\
\text { Asphalt } \\
\text { Content }\end{array}$ & $\begin{array}{c}\text { Initial } \\
\text { Weight } \\
\text { (gram) }\end{array}$ & $\begin{array}{l}\text { Final } \\
\text { Weight } \\
\text { (gram) }\end{array}$ & $\begin{array}{c}\text { Percentage } \\
\text { of Weight } \\
\text { Loss }(\%)\end{array}$ & $\begin{array}{c}\text { Average } \\
(\%)\end{array}$ & $\begin{array}{l}\text { Requirements } \\
\text { Specification }\end{array}$ & Information \\
\hline \multirow{3}{*}{1} & \multirow{3}{*}{$\begin{array}{l}\text { Standard } \\
\text { Asphalt } \\
\text { Mixture }\end{array}$} & \multirow{3}{*}{$6.16 \%$} & 1135.7 & 1089.5 & 4.07 & \multirow{3}{*}{4.04} & & Fulfill \\
\hline & & & 1136.2 & 1091.3 & 3.95 & & & Fulfill \\
\hline & & & 1137.4 & 1090.8 & 4.10 & & & Fulfill \\
\hline \multirow{3}{*}{2} & \multirow{3}{*}{$\begin{array}{c}\text { SBS } \\
\text { modified } \\
\text { asphalt mix }\end{array}$} & \multirow{3}{*}{$6.08 \%$} & 1136.1 & 1099.8 & 3.20 & \multirow{3}{*}{3.14} & & Fulfill \\
\hline & & & 1133.6 & 1097.9 & 3.15 & & $<20 \%$ & Fulfill \\
\hline & & & 1135.8 & 1100.7 & 3.09 & & & Fulfill \\
\hline \multirow{3}{*}{3} & \multirow{3}{*}{$\begin{array}{c}\text { EVA } \\
\text { modified } \\
\text { asphalt mix }\end{array}$} & \multirow{3}{*}{$6.02 \%$} & 1136.4 & 1096.2 & 3.54 & \multirow{3}{*}{3.57} & & Fulfill \\
\hline & & & 1139.7 & 1098.5 & 3.61 & & & Fulfill \\
\hline & & & 1138.3 & 1097.8 & 3.56 & & & Fulfill \\
\hline
\end{tabular}

Based on Table 13, the specimens of standard asphalt AC-WC mixture are $4.04 \%$, SBS modified AC-WC asphalt mixtures are $3.14 \%$, and EVA modified AC-WC asphalt mixtures are $3.57 \%$. The SBS modified AC-WC asphalt mixture was better than the standard AC-WC mixture and the EVA modified AC-WC mixture.

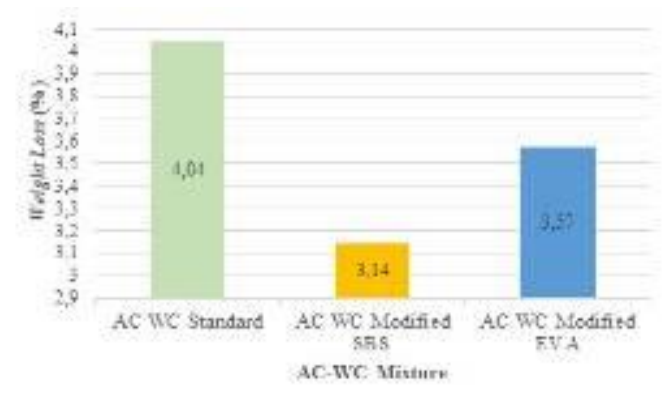

Figure 9. Weight Loss on AC-WC

Tabel 14. Percent of Weight Loss of Hot Rolled Sheet-Wearing Course (HRS-WC)

\begin{tabular}{|c|c|c|c|c|c|c|c|c|}
\hline No. & $\begin{array}{l}\text { Type of } \\
\text { Specimen }\end{array}$ & $\begin{array}{c}\text { Optimum } \\
\text { Asphalt } \\
\text { Content }\end{array}$ & $\begin{array}{l}\text { Initial } \\
\text { Weight } \\
\text { (gram) }\end{array}$ & $\begin{array}{l}\text { Final } \\
\text { Weight } \\
\text { (gram) }\end{array}$ & $\begin{array}{c}\text { Percentage } \\
\text { of Weight } \\
\text { Loss }(\%)\end{array}$ & $\begin{array}{c}\text { Average } \\
(\%)\end{array}$ & $\begin{array}{l}\text { Requirements } \\
\text { Specification }\end{array}$ & Information \\
\hline \multirow{3}{*}{1} & \multirow{3}{*}{$\begin{array}{l}\text { Standard } \\
\text { Asphalt } \\
\text { Mixture }\end{array}$} & \multirow{3}{*}{$7.9 \%$} & 1134.7 & 1090.3 & 3.91 & \multirow{3}{*}{3.91} & \multirow{9}{*}{$<20 \%$} & Fulfill \\
\hline & & & 1135.8 & 1091.6 & 3.89 & & & Fulfill \\
\hline & & & 1135.3 & 1090.8 & 3.92 & & & Fulfill \\
\hline \multirow{3}{*}{2} & \multirow{3}{*}{$\begin{array}{c}\text { SBS } \\
\text { modified } \\
\text { asphalt mix }\end{array}$} & \multirow{3}{*}{$7.88 \%$} & 1136.9 & 1103.2 & 2.96 & \multirow{3}{*}{2.86} & & Fulfill \\
\hline & & & 1134.7 & 1102.5 & 2.84 & & & Fulfill \\
\hline & & & 1135.4 & 1103.8 & 2.78 & & & Fulfill \\
\hline \multirow{3}{*}{3} & \multirow{3}{*}{$\begin{array}{c}\text { EVA } \\
\text { modified } \\
\text { asphalt mix }\end{array}$} & \multirow{3}{*}{$7.83 \%$} & 1134.5 & 1097.3 & 3.28 & \multirow{3}{*}{3.34} & & Fulfill \\
\hline & & & 1135.7 & 1096.6 & 3.44 & & & Fulfill \\
\hline & & & 1133.6 & 1096.1 & 3.31 & & & Fulfill \\
\hline
\end{tabular}


Based on Table 14, the specimen of standard asphalt HRS-WC mixture was $3.91 \%$, SBS modified asphalt HRSWC mixture was $2.86 \%$, and EVA modified asphalt HRS-WC mixture was $3.34 \%$. The SBS modified asphalt HRS-WC mixture performed better than the standard asphalt HRS-WC mixture and the EVA modified asphalt HRS-WC mixture.

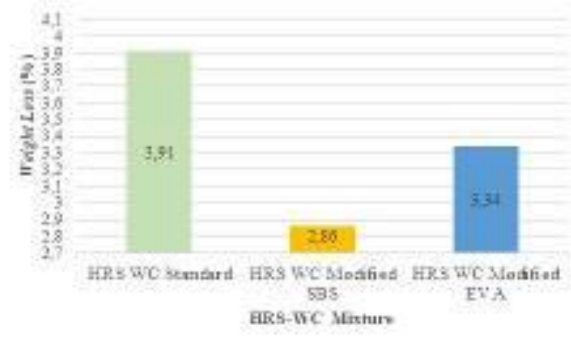

Figure 10. Weight Loss on HRS-WC

\section{CONCLUSION}

\subsection{Based on the Asphalt Pen. 60/70, SBS}

Modified Asphalt, and EVA Modified

Asphalt Characteristics Testing

a. The penetration and ductility test results of SBS modified asphalt are smaller than EVA modified asphalt and asphalt Pen. 60/70. This is because when mixing, SBS modified asphalt hardens faster in cold conditions, so it has stiffer properties, and during ductility testing makes SBS modified asphalt break faster.

b. The results of the flash point and burn point test, EVA modified asphalt were higher than SBS modified asphalt and asphalt Pen. 60/70, so that the EVA modified asphalt was not susceptible to burn at high temperatures.

c. The test results for the softening point of SBS modified asphalt and EVA modified asphalt were higher than asphalt Pen. 60/70, so that modified asphalt (SBS and EVA) had the advantage of being more resistant to high temperatures and would not easily melt where for the requirements of the asphalt resistant to high temperature and does not melt is $\geq 48^{\circ} \mathrm{C}$.

\subsection{Based on Standard Marshall Testing}

a. The KAO values obtained by a mixture of $\mathrm{AC}$ Asphalt Standard, AC asphalt modification $0.5 \%$ EVA and AC asphalt modification $0.5 \%$ SBS were $6.16 \%, 6.02 \%$ and $6.08 \%$, respectively. Meanwhile, the KAO values obtained by a mixture of Standard Asphalt HRS, HRS asphalt modification $0.5 \%$ EVA and HRS asphalt modification $0.5 \%$ SBS were $7.9 \%, 7.835 \%$ and $7.88 \%$, respectively. AC mixture, HRS asphalt modification $0.5 \%$ EVA and AC, HRS asphalt modification $0.5 \%$ SBS have lower KAO values than the AC mixture, HRS Standard Asphalt. So it can be concluded, the addition of EVA and SBS polymers in the asphalt mixture makes the use of asphalt content less than using no polymer.

b. The mixture of AC-WC and HRS-WC asphalt modification $0.5 \%$ SBS is superior in stability and MQ values but has the smallest flow value than the other two mixtures, which causes the mixture to be better at withstanding permanent deformation when receiving past loads without changing shape. The mixture of AC-WC and HRS-WC asphalt modification $0.5 \%$ EVA has a stability and MQ value that is not much different from the mixture of ACWC and HRS-WC asphalt modification $0.5 \%$ SBS but has a higher flow value. The VFA value obtained by the AC-WC mixture and HRS-WC asphalt modification $0.5 \%$ EVA is the smallest from the two other mixtures. The AC and HRS Standard Asphalt mixture had the smallest stability and MQ values, but had the largest VFA and flow values of the other two mixtures.

\subsection{Based on Marshall Immersion Testing}

The Index of Retained Strength (IRS) on the test specimens of the AC-WC asphalt mixture was $92.37 \%$, the AC-WC mixture of SBS modified asphalt was $93.33 \%$, and the AC-WC mixture EVA modified asphalt was $92.74 \%$. The Index of Retained Strength on the test specimen of standard asphalt HRS-WC mixture is $90.25 \%$, the HRS-WC asphalt modified SBS mixture is $91.48 \%$, and the HRS-WC asphalt modified EVA mixture is $90.58 \%$. The sample specimen of SBS modified asphalt mixture produced the best index of retained strength compared to the sample of standard asphalt mixture and EVA modified asphalt mixture. The greater the index of retained strength value, indicating that the sample has better resistance to damage due to the influence of water, temperature and weather. 


\subsection{Based on Cantabro Testing}

The average percent of weight loss (wear value) of the standard AC-WC and HRS-WC asphalt mixture was $4.04 \%$ and $3.91 \%$, the SBS modified asphalt mixture of AC-WC and HRS-WC layers was $3.14 \%$ and $2.86 \%$, then the EVA modified asphalt mixture of AC-WC and HRS-WC layers with an average wear rate of $3.57 \%$ and $3.34 \%$. From the results of the data analysis, it can be seen that the test specimens of the SBS modified asphalt mixture are more resistant to wear than the standard asphalt mixture and EVA modified asphalt mix specimens, because the smaller the percent of weight loss indicates that the mixture is getting stronger because of the lack of mixed particles apart due to the collision event in the spinning machine (Los Angeles), so this causes the high durability of the mixture and the mixture can last for a long time. This provides an illustration of the extent to which the asphalt pavement is resistant to friction between the vehicle wheels and the road surface.

\section{REFERENCES}

[1] Dahlan, A.T. et al. 1997. Improved Design Procedure of Hot Mix Asphalt. Overseas Center, Transport Research Laboratory,
Crowthorne, Berkshire RG 45 6AU, United Kingdom in association with PT Yodya Karya, Indonesia

[2] Bina Marga, 2018. Draft General Specification Division VI Revision 1 for Asphalt Pavement Public Work Department

[3] Mashuri. Astuti, F. and Batti, J. F. 2014. RoadCel-50 on short term aging and durability of the pavement Hot Rolled Sheet Wearing Course (HRS-WC). Journal of Infrastructure Vol. 4 No. 2 December 2014: 103-113

[4] Government of Indonesia 2006. Constiitution 2006 No. 34. State Street Road Republic of Indonesia No 86, State Secretariat: Jakarta

[5] Pradani, N. 2011. Resilient Modulus Performance and Fatigue of Bearing Course using Recycled Materials and StyreneButadiene-Styrene (SBS). Master's Program in Road Management and Engineering of Bandung Institute of Technology, p.134

[6] Sukirman, S.2012. Highway Engineering Second Edition, Chapter 1. Publisher: Bandung, Nova.

[7] Whiteoak, D. 1991. The Shell Bitumen Handbook, Surrey, UK. Shell Bitumen 